\title{
Coherence in Spectroscopy and Modern Physics
}




\section{NATO ADVANCED STUDY INSTITUTES SERIES}

A series of edited volumes comprising multifaceted studies of contemporary scientific issues by some of the best scientific minds in the world, assembled in cooperation with NATO Scientific Affairs Division.

Series B: Physics

RECENT VOLUMES IN THIS SERIES

Volume 29 - Electron-Phonon Interactions and Phase Transitions edited by Tormod Riste

Volume 30 - Many Degrees of Freedom in Field Theory edited by L. Streit

Volume 31 - Many Degrees of Freedom in Particle Theory edited by $\mathrm{H}$. Satz

Volume 32 - Electron and Ion Spectroscopy of Solids edited by L. Fiermans, J. Vennik, and W. Dekeyser

Volume 33 - Microscopic Structure and Dynamics of Liquids edited by J. Dupuy and A. J. Dianoux

Volume 34 - Path Integrals and Their Applications in Quantum, Statistical, and Solid State Physics edited by G. Papadopoulos and J. T. Devreese

Volume 35 - Correlation Functions and Quasiparticle Interactions in Condensed Matter edited by $\mathbf{J}$. Woods Halley

Volume 36 - Strongly Coupled Plasmas edited by Gabor Kalman

Volume 37 - Coherence in Spectroscopy and Modern Physics edited by F. T. Arecchi, R. Bonifacio, and M. O. Scully

Volume 38 - Methods in Medium-Energy and Heavy-Ion Physics edited by K. W. McVoy and W. A. Friedman

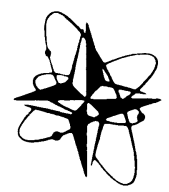

This series is published by an international board of publishers in conjunction with NATO Scientific Affairs Division
A Life Sciences
B Physics
C Mathematical and Physical Sciences
D Behavioral and Social Sciences
E Applied Sciences

Plenum Publishing Corporation
D. Reidel Publishing Company Dordrecht and Boston

Sijthoff International Publishing Company

Noordhoff International Publishing Leiden 


\section{Coherence \\ in Spectroscopy \\ and Modern Physics}

Edited by

F.T. Arecchi

University of Florence

and National Institute of Optics

Florence, Italy

R. Bonifacio

University of Milan

Milan, Italy

and

M.O. Scully

University of Arizona

Tucson, Arizona 


\section{Library of Congress Cataloging in Publication Data}

Nato Advanced Study Institute on Coherence in Spectroscopy and Modern Physics, Versilia, Italy, 1977.

Coherence in spectroscopy and modern physics.

(NATO advanced study institutes series: Series B, Physics; v. 37)

"Proceedings of the NATO Advanced Study Institute on Coherence in Spectroscopy and Modern Physics held at Villa LePianore, Versilia, Italy, July 17-30, 1977."

Includes index.

1. Coherence (Optics) - Congresses. 2. Spectrum analysis - Congresses. 3. Physics - Congresses. I. Arecchi, F. T. II. Bonifacio, R. III. Scully, Marlan Orvil, 1939IV. Title. V. Series.

QC476.C6N37 1977

536

78-14474

ISBN-13: 978-1-4613-2873-5

e-ISBN-13: 978-1-4613-2871-1

DOI: $10.1007 / 978-1-4613-2871-1$

Proceedings of the NATO Advanced Study Institute on Coherence in Spectroscopy and Modern Physics held at Villa LePianore, Versilia, Italy, July 17-30, 1977

๑ 1978 Plenum Press, New York Softcover reprint of the hardcover 1st edition 1978

A Division of Plenum Publishing Corporation

227 West 17th Street, New York, N.Y. 10011

All rights reserved

No part of this book may be reproduced, stored in a retrieval system, or transmitted, in any form or by any means, electronic, mechanical, photocopying, microfilming, recording, or otherwise, without permission from the Publisher 


\section{PREFACE}

This volume contains the lectures and seminars presented at the NATO Advanced Study Institute on "Coherence in Spectroscopy and Modern Physics," the seventh course of the International School of Quantum Electronics, affiliated with the "Ettore Majorana" Centre for Scientific Culture, Erice, Sicily. The Institute was held at Villa LePianore (Lucca), Versilia, Italy, July 17-30, 1977.

The Internationa1 School of Quantum Electronics was started in 1970 with the aim of providing instruction for young researchers and advanced students already engaged in the area of quantum electronics or wishing to switch to this area from a different background. From the outset the School has been under the direction of Prof. F. T. Arecchi, then at the University of Pavia, now at the University of Florence, and Dr. D. Roess of Siemens, Munich. Each year the Directors choose a subject of particular interest, alternating fundamental topics with technological ones, and ask colleagues specifically competent in a given area to take the scientific responsibility for that course.

The past courses were devoted to the following themes:

1971: "Physical and Technical Measurements with Lasers"

1972: "Nonlinear Optics and Short Pulses"

1973: "Laser Frontiers: Short Wavelength and High Powers"

1974: "Cooperative phenomena in Multicomponent Systems"

1975: "Molecular Spectroscopy and Photochemistry with Lasers"

1976: "Coherent Optical Engineering"

The purpose of the present course was to provide instruction in the field of modern physics and spectroscopy as it is influenced by coherent quantum-mechanical phenomena. It is generally acknowledged that this rapidly developing field of research, besides offering an intellectual challenge, has had a significant impact on modern technology.

Prof. R. Bonifacio, University of Milan, and Prof. M. 0. Scully, University of Arizona, undertook the scientific direction of the 
present course, selecting the specific topics and lectures.

The lectures collected in this volume cover the basic concepts and principles of coherence in quantum optics as well as in other areas of physics together with the most promising applications.

We wish to express our appreciation to the NATO Scientific Affairs Division, whose financial support made this Institute possible. We also acknowledge the contribution of the following:

CISE

IBM Italia

Philips, Eindhoven

Siemens, Munich

We finally thank the secretaries of Divisione Elettronica Quantistica, CISE, Mrs. G. Ravini and Miss M. Oriani, for their valuable assistance in the organization of the Institute and in the preparation of these proceedings, and Miss A. Camnasio of Servizio Documentazione, CISE, and again Mrs. G. Ravini for their assistance during the course itself.

F. T. Arecchi

R. Bonifacio

M. O. Scul1y 
CONTENTS

A - Basic Tools

Coherence in Spectroscopy .. . . . . . . . . . . . . 1

A. Kastler

Coherence in Synergetics . . . . . . . . . . . . . 21

H. Haken

$$
\text { B - Coherence in Spin-like Systems }
$$

Coherent Optical Transients . . . . . . . . . . . 41

R. G. Brewer

Mean Field Theory of Optical Bistability and Resonance

Fluorescence ................ 85

L. A. Lugiato and R. Bonifacio

Optical Bistability and Differential Gain . . . . . . . . 111

H. M. Gibbs, S. L. McCa11, and T. N. C. Venkatesan

Superfluorescence Experiments . . . . . . . . . . . 121

H. M. Gibbs

Coherent Two-Photon Amplication in an Inverted Medium . . . . 131

L. M. Narducci, L. G. Johnson, E. J. Seibert, and

W. W. Eidson

$$
\text { C - Coherence in Atomic Spectroscopy }
$$

High Resolution Spectroscopy in Atomic Rydberg States . . . 151

S. Haroche

The Method of Separated Fields in Optics . . . . . . . . 173

V. P. Chebotayev

Coherence in Beam-Foil Spectroscopy . . . . . . . . . . 195

H. J. Andrä

Magnetic Resonances in Sodium Vapours Oriented by a C. W. Dye

Laser . . . . . . . . . . . . . . 219

G. Alzetta 
D - Applications of Coherence to Physical Systems

Superconductivity and Quantum Optics . . . . . . . . . 231

A. Di Rienzo, D. Rogovin, M. Scully, R. Bonifacio,

L. Lugiato, and M. Milani

Surface Plasmon Effects on Molecular Decay Processes near

Metallic Interfaces . . . . . . . . . . 261

H. Morawitz

Introduction to Picosecond Spectroscopy . . . . . . . . . 301

B. Bosacchi

Picosecond Spectroscopy and Solid State Physics . . . . . 305

B. Bosacchi

Coherent Effects in Picosecond Spectroscopy . . . . . . . 329

A. Laubereau and W. Kaiser

Nonthermal Microwave Resonances in Living Cells . . . . . . 347

F. Keilmann

$$
\text { E - New Laser Sources }
$$

The Free Electron Laser: A Single Particle Classical Model • • 361 A. Bambini and A. Renieri

Frequency Tunability and High Speed Modulation of Far Infrared Optically Pumped Lasers by Stark Effect . . . . . 381

F. Strumia

Index 